Insgesamt zeichnet Schulz ein sehr differenziertes und praxisnahes Gesamtbild der parlamentarischen Beteiligungswege in europäischen Angelegenheiten. Danach sind die formalen Verfahren mit dem durch Gewaltenverschränkung geprägten politischen System der Bundesrepublik wenig kompatibel und insofern in der Praxis gegenüber den spezifischen informalen Mitwirkungswegen von Mehrheits- und Oppositionsfraktionen kaum von Bedeutung.

Ein Schwachpunkt der Studie liegt in der speziell zu Beginn der Arbeit recht losen Anbindung an bestehende Forschungsergebnisse. Analysen der praktischen Mitwirkung des Deutschen Bundestags in europäischen Angelegenheiten, die später durchaus Erwähnung finden (zum Beispiel S. 51), werden einleitend kaum diskutiert. Die vierseitige Darlegung des Forschungsstandes erscheint somit als Pflichtübung. Sie wird weder zur Präzisierung zentraler Konzepte genutzt (Mitwirkung), noch dient sie der Generierung plausibler Erwartungen hinsichtlich der akteursspezifischen Ziele und Mechanismen der Mitwirkung sowie der entsprechenden Instrumente. Hier hätte die Studie durch eine einleitende, von Beginn an entlang des neuen Dualismus strukturierte Diskussion möglicher Mitwirkungswege an Stringenz gewonnen. So mag es zum Beispiel angesichts vielfältiger Ausführungen über die für parlamentarische Systeme typische Verzahnung von Regierung und Parlamentsmehrheit nicht gerade überraschen, dass die Einflussnahme in europäischen Angelegenheiten seitens der Mehrheitsfraktionen im ,intra-party-mode' erfolgt, zumal der Autor selbst betont, dass das „Konzept einer informalen Mitsteuerung allgemein anerkannt ist“ (S. 163). Ebenso verwundert es angesichts teilweise auch genannter Ausführungen von Arthur Benz sowie Katrin Auel kaum, dass der Opposition zur Mitwirkung trotz aller damit verbundenen Schwierigkeiten in erster Linie der Weg der öffentlichen Kritik des Regierungshandelns offen steht. Schulz' Andeutung, dass bislang keine „klare[n] Ideen - Konzepte - davon bestünden, wie und auf welchen Wegen der Bundestag in europäischen Angelegenheiten mitwirkt" (S. 19), ist in Anbetracht dessen kritisch zu hinterfragen.

Ungeachtet dieser Kritik ist die Studie insgesamt lesenswert. Sie liefert eine umfangreiche Deskription, die an Tiefenschärfe und Aktualität kaum Wünsche offen lässt. Damit leistet Schulz einen wichtigen Beitrag zur empirischen Analyse der tatsächlichen Mitwirkung des Deutschen Bundestags im europäischen Mehrebenensystem.

Lisa Helene Anders

\title{
Europarecht - grundlegende Kommentierung und engagierte Positionen
}

Schwarze, Jürgen (Hrsg.): EU-Kommentar, 3. Auflage, Nomos Verlagsgesellschaft, Helbing Lichtenhahn Verlag, facultas.wuv Verlag, Baden-Baden / Basel / Wien 2012, 3019 Seiten, $€ 225$,-

Schwarze, Jürgen: Europarecht, Strukturen, Dimensionen und Wandlungen des Rechts der Europäischen Union, Nomos Verlagsgesellschaft, Baden-Baden 2012, 1151 Seiten, € 128,-.

Einen deutlichen Gewinn an Demokratie brachte der EU Ende 2009 der Reformvertrag von Lissabon. Das Europäische Parlament - das Bundesverfassungsgericht verneinte im Lissabon-Urteil vom 30. Juni 2009 abschätzig die Qualität einer Volksvertretung und stellte es 
am 9. November 2011 im Urteil zur Fünfprozentklausel Kommunalvertretungen gleich wurde wesentlich gestärkt: Es ist nun auch für die Gesetze zu Polizeilicher und Justizieller Zusammenarbeit zuständig; die Rechte zur Wahl der Kommission und Misstrauensvoten wurden ausgebaut. Die Europäische Bürgerinitiative schuf ein mittlerweile schon fast 20-mal genutztes Instrument bürgerschaftlicher Einmischung, wozu es auf Bundesebene keine Entsprechung gibt. Natürlichen und juristischen Personen wurden Nichtigkeitsklagen auch gegen das Handeln der Union und Rechtsakte mit Verordnungscharakter ermöglicht. Die Grundrechtecharta wurde Teil des Primärrechts; etwa bei der mittelbaren Diskriminierung der Frauen im Arbeitsleben vermittelt die EuGH-Rechtsprechung einen höheren Grundrechtsstandard als Grundgesetz und Bundesverfassungsgericht. Spannend wird, wie sich nach dem Beitritt der EU zur Europäischen Menschenrechtskonvention die Konkurrenz von EuGH und Europäischem Gerichtshof für Menschenrechte darstellt.

Die Verträge wurden vereinheitlicht, nur noch die EU ist völkerrechtlicher Mitspieler. Doch bleibt es bei zwei, inhaltlich voneinander abhängigen Verträgen, dem über die Europäische Union (EUV) und dem Vertrag über die Arbeitsweise der Europäischen Union (AEUV). Der einheitliche Verfassungsvertrag scheiterte nach Volksabstimmungen in Frankreich und den Niederlanden, die wenig mit Europa, doch viel mit innenpolitischen Querelen zu tun hatten. Lissabon brachte „nur“ eine Reform des Vertrags von Nizza. Der „ausgesprochen komplexe“ Vertrag „spiegelt damit die lange, durch vielfältige Kompromisse geprägte Entstehungsgeschichte wider“, so Jürgen Schwarze im Vorwort. Es bleibt bei der oft aus nationalem Sonderinteresse geborenen inhaltlichen Unübersichtlichkeit bis hin zu zulässigen Beihilfen im lange nicht mehr bestehenden Zonenrandgebiet (Art. 107 Abs. 2 lit. c AEUV), die die Bundesregierung vergeblich auf die östlichen Bundesländer ausdehnen wollte.

Der von Jürgen Schwarze, einem versierten Europarechtler, verantwortete Kommentar, woran neben den Mitherausgebern Armin Hatje und Johann Schoo 48 bestens ausgewiesene Wissenschaftler mitwirkten - nur acht Frauen, von 14 wissenschaftlichen Mitarbeitern sieben -, ist ein kaum verzichtbarer Wegweiser durch das Dickicht der Vertragsstrukturen. Leider fehlt wie in den Vorauflagen der Vertrag über die Europäische Atomgemeinschaft, von der Kommentierung ganz zu schweigen. Zweifelhaft ist, wenn die Einleitung unter Bezugnahme auf Art. 17 Abs. 5 EUV suggeriert, ein Drittel der Kommissionsmitglieder werde ab November 2014 wegfallen. Tatsächlich behält Irland, wie im Kommentar erläutert, gemäß den Konzessionen des Europäischen Rats vom 11./12. Dezember 2008 vor der zweiten Volksabstimmung weiter seinen Kommissar, was dann für alle Mitgliedstaaten gilt, zumal Beschlüsse über die Struktur der Kommission einstimmig sein müssen. Die Kommission wird also nicht verkleinert: Es muss ihre Handlungsfähigkeit nicht beeinträchtigen, wie die großen Bundeskabinette Konrad Adenauers bewiesen.

Gegenüber der Vorauflage, in der auf den Lissabon-Vertrag verwiesen wurde, er aber nicht Grundlage der Kommentierung sein konnte, ist es eine grundlegende Neuauflage. Die Komplexität des Vertragswerks wird in verständlicher Form erklärt. Dazu trägt jedenfalls bei umfangreichen Bestimmungen der Aufbau der Kommentierung bei mit der Literaturübersicht, allgemeinen Darstellung, dem Hinweis zur Rechtsentwicklung und / oder Anwendungsbereichen und Begriffsbestimmung. Die eigentliche Kommentierung enthält umfangreiche Angaben zur Literatur, zu Sekundärrecht und Rechtsprechung, gegebenenfalls als mehrseitiger Anhang wie zu Art. 113 AEUV mit den Entscheidungen zur 6. Mehrwertsteuer-Richtlinie. 
Wie zuvor auf den erwarteten Lissabon-Vertrag verwiesen wurde, gibt es nun eine Orientierungshilfe zur Stabilitätsunion. Insbesondere wird im Anhang der am 25. März 2012 von 25 Mitgliedstaaten - ohne das Vereinigte Königreich und Tschechien - unterzeichnete Vertrag über Stabilität, Koordinierung und Steuerung in der Wirtschafts- und Währungsunion abgedruckt. Ebenfalls sind dort die wichtigen Protokolle enthalten, unter anderem zur Grundrechtecharta das Opt-out des Vereinigten Königreichs und Polens im Protokoll 30 und zusätzlich Warschaus Erklärung 61 zum Lissabon-Vertrag für die Bereiche der öffentlichen Sittlichkeit, des Familienrechts und der Achtung der moralischen Unversehrtheit, die an der Weichsel und Themse nicht gelten. Es wird sich zeigen, ob das angesichts der umfangreichen Grundrechtsrechtsprechung des EuGH praktische Bedeutung haben wird. Hinzu kommen im Anhang die einschlägigen Regeln über die Gerichtsorganisation und Verfahrensordnungen der europäischen Gerichte mit praktischen Hinweisen für die Parteien, Prozessvertreter und vorlegenden nationalen Gerichte. Es gibt ein ausführliches Stichwortverzeichnis, doch keine Konkordanztabelle zu den früheren Verträgen.

Bei der so konstituierten Union wird es nicht bleiben. In Teilbereichen - jüngst zur Einführung der Finanztransaktionssteuer - wird es zu verstärkter Zusammenarbeit von Mitgliedstaaten kommen, wie in der Kommentierung zu Art. 326 ff. AEUV ausführlich erläutert. Vor allem werden die Euro-Staaten einen wirtschaftlichen, in der Folge politischen Kern bilden. Der neue Absatz 3 zu Art. 136 AEUV, als Grundlage des Europäischen Stabilitätsmechanismus (ESM) im vereinfachten Verfahren einstimmig verabschiedet, ist ein Symbol; er wurde in die Kommentierung, doch nicht in den Vertragswortlaut aufgenommen. Derweil London aus der Union, zumindest in der heutigen Verfassung hinausstrebt, wollen andere, etwa Polen, in den Kreis der Vereinigungsapologeten. Auch Deutschland, eine weitere Regierung Merkel sowie das Bundesverfassungsgericht werden den Weg mitgehen.

Daher trifft es sich gut, den von Jürgen Schwarze edierten Sammelband eigener Aufsätze zu Strukturen, Dimensionen und Wandlungen des Rechts der Europäischen Union mitzubetrachten: 47 Aufsätze und zusammenfassende Würdigungen von Projektstudien von 1983 bis 2011, ob der Aktualität vor allem aus den letzten gut zehn Jahren. Sie sind in zehn Komplexe gegliedert, namentlich zum Konzept des Unionsrechts, dem Staats- und Verfassungsrecht in Europa, vor allem dem Verhältnis nationalen Verfassungs- und europäischen Rechts, zur Kompetenzverteilung und institutionellen Ordnung, zum Grundrechtsschutz in der EU, zur Europäischen Verfassungsreform anhand der Voraussetzungen und Perspektiven einer Verfassung, des damaligen Verfassungsvertrags und Lissabon-Vertrags, zum nationalen Verwaltungsrecht und -rechtsschutz in vergleichender und europäischer Perspektive, zur Entstehung und Entwicklung eines europäischen Verwaltungsrechts, zum Rechtsschutz in der EU, zu den Medien und dem Schutz des geistigen Eigentums in europäischer Perspektive, endlich zum Europäischen Wirtschafts- und Wettbewerbsrecht, vor allem Wirtschaftsverfassung, Kartell- und Beihilferecht, Fusionskontrolle und Recht der öffentlichen Aufträge.

Die Beiträge zeigen ein Gesamtbild der wesentlichen Probleme im Unionsrecht. Erstaunlich ist, wie strukturgezielt die Aufsätze geschrieben wurden. Denn „wer das Europarecht genauer kennenlernen will, muss sich zunächst mit seinen Strukturmerkmalen vertraut machen", so Schwarze in der Einführung. Zentral ist die auch vom Bundesverfassungsgericht im Nachvollzug des COSTA / ENEL-Urteils des EuGH schon am 18. Oktober 1967 anerkannte Eigenständigkeit des Unionsrechts gegenüber dem der Mitgliedstaaten und dem Völkerrecht. Im Vorrang vor deren Recht gewährt es dank unmittelbarer Wirkung dem 
einzelnen einklagbare Rechte aus dem Primär- und Sekundärrecht gegenüber abweichendem Handeln von Mitgliedstaaten. Diese Strukturmerkmale des Europarechts als Konstante europäischer Rechtsbildung geben der fortschreitenden Integration Form und Halt, so Jürgen Schwarze.

Zudem hat sich das ursprünglich für den Gemeinsamen Markt konzipierte Recht „über die Jahrzehnte in immer weitere Dimensionen ausgedehnt“. Die heutige Reichweite betreffe fast alle Bereiche, „,in denen über die grundsätzlichen Gestaltungsprobleme unserer wirtschaftlichen und politischen Ordnung entschieden wird“, gekennzeichnet von ungeheurer Schnelligkeit und Wandlungsfähigkeit. Schon für Walter Hallstein sollte das Europarecht „als Mittel zur Herbeiführung der europäischen Integration dienen“. Mit wachsender Regelungstiefe schufen für Schwarze die Verträge eine Verfassungsgrundlage der heutigen Union. Im Anschluss hätten sich Ansätze für ein europäisches Verwaltungsrecht zum Vollzug der gemeinsamen Rechtsregeln entwickelt. Noch immer sei die Ordnung und Gestaltung der Wirtschaft zentrale Aufgabe des Unionsrechts. Die ständigen Veränderungen seien aber eine Grundanforderung für seine Flexibilität, zumal vor dem Hintergrund sich schnell wandelnder internationaler Verhältnisse. Hinzu komme die räumliche Ausdehnung auf immer mehr Mitgliedstaaten, deren Zusammenführung Kompromisse, also Flexibilität brauche.

Die vorgelegten Aufsätze spiegeln die Wandlungen wider, auch über die Jahre und Jahrzehnte. Sie zeigen den Autor als „überzeugten Europäer“, schon das ein Grund für seine negative Bewertung des Lissabon-Vertrag-Urteils: „Das Gebot der Hinwendung zu Europa spiegelt das Grundgesetz deutlich wider, wenn es die deutsche Politik und das deutsche Recht auf Europa und die europäische Einigung verpflichtet." Dem sei Karlsruhe nicht gerecht geworden; auch die Politik und (ver-)öffentlich(t)e Meinung trage dem nicht immer Rechnung; sie stünden im Gegensatz zu Europas Errungenschaften und seinem Recht.

Dieses Buch will einen Beitrag dazu leisten, dem Europarecht die gebührende Berücksichtigung auch in der öffentlichen Debatte zu geben. Als klare europapolitische Stellungnahme steht es neben der von Jürgen Schwarze in dritter Auflage immer weiter verfeinerten Analyse der Vertragsnormen. Der Kommentar wird für einige Jahre in Wissenschaft und Praxis eine Grundlage der europarechtlichen und -politischen Diskussionen sein. Die auch im Sammelband erläuterten Wandlungsprozesse und die davon ausgehende Dynamik der (welt-)wirtschaftlichen politischen Entwicklungen und der Rechtsprechung des EuGH, die in den nächsten fünf Jahren wohl anstehende Revision des Lissabon-Vertrags - möglicherweise ein neuer Vertrag - werden in geraumer Zeit eine 4. Auflage erfordern. Denn Europas Integration ist nicht am Ende. Sie wird auch die Karlsruher Bremsklötze überwinden. Darin sieht Jürgen Schwarze die einzige Chance für die in den Verträgen grundgelegte Wahrung der Menschenwürde, Freiheit, Demokratie, Gleichheit, Rechtsstaatlichkeit, für den Frieden und das Wohlergehen der Völker in einem Raum der Freiheit, der Sicherheit und des Rechts. Durch die Darstellung der Grundlagen und Probleme der Union leisten die beiden Bücher einen wichtigen Beitrag und sind für die Verantwortlichen in Politik und Verwaltung wieder eine Entscheidungshilfe in allen europarechtlichen Fragen. 